\title{
IncRNA TPTEP1 inhibits stemness and radioresistance of glioma through miR-106a-5p-mediated P38 MAPK signaling
}

\author{
TING TANG $^{1}$, LING-XING WANG ${ }^{1}$, MEI-LI YANG ${ }^{1}$ and RONG-MOU ZHANG ${ }^{2}$ \\ Departments of ${ }^{1}$ Neurology and ${ }^{2}$ Spine Surgery, The Second Affiliated Hospital of \\ Fujian Medical University, Quanzhou, Fujian 362000, P.R. China
}

Received May 12, 2020; Accepted September 1, 2020

DOI: $10.3892 / \mathrm{mmr} .2020 .11542$

\begin{abstract}
Glioma is diagnosed as the most common intracranial malignant tumor. Cancer stem cells determine stemness and radioresistance, and may facilitate glioma recurrence. The present study aimed to investigate whether the long non-coding RNA (lncRNA) transmembrane phosphatase with tensin homology pseudogene 1 (TPTEP1) regulated cell stemness and radioresistance of glioma, and determine the underlying molecular mechanism of TPTEP1 in the modulation of glioma progression. Cell and molecular biology techniques were applied for investigating the role of TPTEP1 in glioma cell lines, animal model, and clinical samples. The results demonstrated that TPTEP1 attenuated stemness and radioresistance of glioma both in vitro and in vivo. In addition, TPTEP1 augmented MAPK14 expression by competitively interacting with microRNA (miR)-106a-5p, thus activating the P38 MAPK signaling pathway, and suppressing glioma stemness and radioresistance. TPTEP1 functionally bound to miR-106a-5p, which formed a reciprocal regulatory loop to stimulate the P38 MAPK signaling pathway. Low TPTEP1 expression levels were detected in high-grade glioma tissues compared with low-grade glioma tissues, and were positively associated with poor prognosis of patients with glioma. Furthermore, analysis using data from The Cancer Genome Atlas database confirmed the molecular mechanism and biological significance of dysregulation of TPTEP1 in glioma
\end{abstract}

Correspondence to: Dr Rong-Mou Zhang, Department of Spine Surgery, The Second Affiliated Hospital of Fujian Medical University, 34 Zhongshan North Road, Quanzhou, Fujian 362000, P.R. China

E-mail: zhangrongmou123@163.com

Abbreviations: ceRNAs, competing endogenous RNAs; CSCs, cancer stem cells; GBM, glioblastoma multiforme; GSEA, gene set enrichment analysis; LGG, brain lower grade glioma; IncRNAs, long non-coding RNAs; ISH, in situ hybridization; RIP, RNA immunoprecipitation; RISC, RNA-induced silencing complexes

Key words: transmembrane phosphatase with tensin homology pseudogene 1, microRNA-106a-5p, MAPK14, stemness, radioresistance, glioma progression. Taken together, the results of the present study suggest that TPTEP1 may be applied as a diagnostic and prognostic indicator for glioma, and may be an alternative target for the treatment of glioma.

\section{Introduction}

Glioma, which is characterized at a high rate of local recurrence, is located in the frontal lobe, temporal, parietal, occipital lobe, or deeper structures and accounts for $~ 80 \%$ of malignant primary brain and CNS tumors in recent years (1-3). Local recurrence and resistance to therapies are the predictors for glioma prognosis, and approaches aimed at eliminating cancer recurrence and alleviating therapeutic resistance are potential strategies for glioma treatment (4). Cancer stem cells (CSCs) are regarded as the main contributor to cancer recurrence, and are known to survive following radiotherapy and chemotherapy of different types of cancer, such as glioma, liver cancer and breast cancer (5-8). Thus, targeting CSCs may be an effective therapy in improving the prognosis of patients with glioma.

Long non-coding RNAs (lncRNAs) exert important functions in biological processes, such as chromatin remodeling, transcriptional activation and interference, RNA processing and mRNA translation (9), thereby modulating cell proliferation and development, cell cycle, cell differentiation, stress responses and the occurrence of several diseases in humans and animals, such as cancer, cardiovascular disease and Alzheimer's disease (10). Previous studies have reported that lncRNAs have crucial roles in modulating cancer stemness (11-15). For example, the 1ncRNA, TP73-AS1 has been demonstrated to promote tumor aggressiveness and temozolomide resistance in $\mathrm{CSCs}$, which confers a poor prognosis in glioblastoma multiforme (GBM) (16). Another IncRNA, Linc00152, has been reported to enhance the malignant behavior of CSCs via the microRNA (miRNA/miR)-103a-3p/FEZ family zinc finger $1 / \mathrm{Cell}$ Division Cycle 25A axis, thereby upregulating the PI3K/AKT signaling pathway (17). Furthermore, nano complex targeting metastasis associated lung adenocarcinoma transcript 1 may lower the stemness of GBM cells and improve the sensitivity of GBM cells to the current first-line therapy of GBM (18). In addition, lncRNAs have also been indicated to participate in the regulation of glioma radioresistance (19). The upregulation of the lncRNA HIF1A antisense RNA 2 and lncRNA 
small nucleolar RNA host gene 18 was demonstrated to promote glioma cell radioresistance $(20,21)$. However, the function of the lncRNA transmembrane phosphatase with tensin homology pseudogene 1 (TPTEP1) in glioma currently remains unknown, and any potential influence of the lncRNA TPTEP1 in the regulation of stemness and radioresistance of glioma is yet to be determined.

The present study aimed to investigate the impact of lncRNA TPTEP1 on glioma and determine the underlying molecular mechanism of TPTEP1 on the regulation of glioma progression. The results demonstrated that TPTEP1 upregulated MAPK14 (P38) by competitively interacting with miR-106a-5p, and inhibited glioma cell stemness and radioresistance both in vitro and in vivo. Notably, TPTEP1/miR-106a-5p was demonstrated to form a reciprocal regulatory circuit to impede glioma development. Furthermore, low TPTEP1 expression levels were detected in high-grade glioma tissues compared with low-grade glioma tissues, and were associated with poor overall survival time of patients with glioma.

\section{Materials and methods}

Cell culture. Human low malignancy grade SHG44 cells and high malignancy grade U-251MG cells were purchased from the Cell Bank of Type Culture Collection of the Chinese Academy of Sciences. Cells were maintained in DMEM medium supplemented with $10 \%$ fetal bovine serum (HyClone; GE Healthcare) and $1 \%$ penicillin/streptomycin, at $37^{\circ} \mathrm{C}$ in a humidified incubator with $5 \% \mathrm{CO}_{2}$. The cell lines were verified to be free of mycoplasma contamination.

Cell transfection. Specific short hairpin (sh)RNAs against TPTEP1 (sh-TPTEP1), lncRNA TPTEP1 sequence (TPTEP1) and their corresponding negative control (NC) sequences were purchased from GeneChem, Inc. Lentivirus particles were subsequently constructed and amplified, and polybrene reagent (GeneChem, Inc.) was used for transfection (MOI: $10,37^{\circ} \mathrm{C}$ for $\left.12 \mathrm{~h}\right)$ in glioma cells $\left(1 \times 10^{4}\right)$. Plasmids were purchased from Vigene Bioscience, Inc. Mimics (5'-AAA AGUGCUUACAGUGCAGGUAG-3'), mimics control (5'-UUUGUACUACACAAAAGUACUG-3'), inhibitors (5'-CUACCUGCACUGUAAGCACUUUU-3'), inhibitors control (5'-CAGUACUUUUGUGUAGUACAAA 3'), small interfering (si)RNAs (5'-ACAGCAUCGAAGUAAGAGAUC UCUUACUUCGAUGCUGU-3') and siRNA control (5'-UUU GUACUACACAAAAGUACUG-3') were purchased from Guangzhou RiboBio Co., Ltd. Lipofectamine ${ }^{\mathrm{TM}} 2000$ reagent (Invitrogen; Thermo Fisher Scientific, Inc.) was to transfect $4 \mu \mathrm{g}$ plasmids (oe-TPTEP1; GeneChem, Inc.), $50 \mathrm{nM}$ siRNAs (si-TPTEP1), $100 \mathrm{nM}$ inhibitors and/or $50 \mathrm{nM}$ mimics into glioma cells $\left(1 \times 10^{5}\right)$. The cells were subjected to subsequent experiments $48-72 \mathrm{~h}$ post-transfection.

Tumorspheres formation assay. Glioma cells $\left(5 \times 10^{3}\right)$ in the exponential growth phase were seeded into ultra-low adherence 6-well plates, and propagated in serum-free DMEM/Ham's F-12 medium supplemented with $20 \mathrm{ng} / \mathrm{ml}$ epidermal growth factor, $20 \mathrm{ng} / \mathrm{ml}$ fibroblast growth factor and $2 \%$ B27 supplement (for supporting neuronal cell proliferation), all purchased from Invitrogen; Thermo Fisher Scientific, Inc.. Following cell culture for $2-3$ weeks at $37^{\circ} \mathrm{C}$, the resultant tumorspheres were imaged under a light microscope (x100 magnification) and subjected to further analysis.

MTT assay. Glioma cells $\left(1 \times 10^{3}\right)$ in the exponential growth phase were seeded into 96 -well plates and incubated with MTT (Invitrogen; Thermo Fisher Scientific, Inc.) at $37^{\circ} \mathrm{C}$ for $4 \mathrm{~h}$. Following the MTT incubation, the purple formazan crystals were dissolved using dimethyl sulfoxide (Invitrogen; Thermo Fisher Scientific, Inc.) and viability was subsequently analyzed at $570 \mathrm{~nm}$ using a Multiskan ${ }^{\mathrm{TM}}$ FC (Thermo Fisher Scientific, Inc.).

Immunofluorescence. Cells $\left(5 \times 10^{4}\right)$ were seeded onto glass slides and allowed to adhere. Cells were subsequently fixed with $4 \%$ paraformaldehyde diluted with PBS for $15 \mathrm{~min}$ at room temperature, permeabilized with $0.2 \%$ Triton X-100 diluted with PBS for $20 \mathrm{~min}$ at room temperature and blocked with $10 \%$ goat serum diluted with PBS (Fuzhou Maixin Biotech Co., Ltd.) for $30 \mathrm{~min}$ at $37^{\circ} \mathrm{C}$. Cells were incubated against the following antibodies: CD133 (1:500; ProteinTech Group, Inc.; cat. no. 66666-1-Ig) or $\gamma$-histone H2AX (H2AX; 1:500; Abcam; cat. no. ab2893) at $4^{\circ} \mathrm{C}$ for $16 \mathrm{~h}$, and PE-conjugated secondary antibody at $37^{\circ} \mathrm{C}$ for $1 \mathrm{~h}(1: 200$; Invitrogen; Thermo Fisher Scientific, Inc.; cat. no. P-2771MP.) diluted with PBS. Cells were subsequently co-stained with DAPI (Sigma-Aldrich; Merck $\mathrm{KGaG}$ ) for $10 \mathrm{~min}$ at room temperature and observed under a fluorescence or confocal microscope (x400 magnification).

Ionizing radiation treatment. Cells $\left(5 \times 10^{6}\right)$ were cultured at with DMEM in $10 \mathrm{~cm}$ plates and irradiated with a $6 \mathrm{MV}$ linear accelerator (Siemens AG). During irradiation for 1-2 min, cells were treated $50 \mathrm{~cm}$ from the source of radiation, with a dose of 4 Gy at room temperature.

Reverse transcription-quantitative PCR (RT-qPCR). Total RNA was extracted from glioma cells using TRIzol ${ }^{\circledR}$ reagent (Invitrogen; Thermo Fisher Scientific, Inc.) and was reverse-transcribed into cDNA using the reverse transcriptase kit (cat. no. D350A, Takara Bio, Inc.), according to the manufacturer's protocols. qPCR was subsequently performed using the SYBR Premix Ex Taq ${ }^{\text {TM }}$ II kit (cat. no. DRR081, Takara Bio, Inc.), on an ABI ViiA 7 sequence detection system (Applied Biosystems; Thermo Fisher Scientific, Inc.), according to the manufacturer's protocols. The following primer sequences were used for qPCR: Hsa-miR-106a-5p primer, 5'-AAAAGT GCTTACAGTGCAGGTAG-3'; TPTEP1 forward, 5'-CTG GGAGAAGTGCCCTTGC-3' and reverse, 5'-CACCTCATC AGTCATTTGCTCA-3'; U6 forward, 5'-CTCGCTTCGGCA GCACA-3' and reverse, 5'-AACGCTTCACGAATTTGC GT-3'; and GAPDH forward, 5'-GCAAATTCCATGGCA CCGT-3' and reverse, 5'-TCGCCCCACTTGATTTTGG-3'. The following thermocycling conditions were used for qPCR: Initial denaturation at $95^{\circ} \mathrm{C}$ for $10 \mathrm{sec}$, followed by 40 cycles of denaturation at $94^{\circ} \mathrm{C}$ for $5 \mathrm{sec}$ and annealing/extension at $60^{\circ} \mathrm{C}$ for $30 \mathrm{sec}$. Relative gene expression levels were calculated using the $2^{-\Delta \Delta \mathrm{Cq}}$ method (22) and normalized to the internal reference genes U6 or GAPDH. 
Western blotting. Glioma cells in the exponential growth phase were lysed and collected using RIPA lysis buffer (Beyotime Institute of Biotechnology). Total protein was quantified using the bicinchoninic acid assay protein kit (cat. no. 23227, Pierce; Thermo Fisher Scientific, Inc.), according to the manufacturer's protocol. A total of 30-50 $\mu \mathrm{g}$ proteins were loaded to each well and separated by $10 \%$ SDS-PAGE gel. The separated proteins were transferred onto polyvinylidene difluoride membranes (Thermo Fisher Scientific, Inc.) and blocked with $3 \%$ bovine serum albumin (Invitrogen; Thermo Fisher Scientific, Inc.) at $37^{\circ} \mathrm{C}$ for $1 \mathrm{~h}$. The membranes were incubated with primary antibodies against: p38 MAPK (1:2,000; ProteinTech Group, Inc.; cat. no. 66234-1-Ig), phosphorylated (p-)p38 MAPK (1:1,000; Abcam; cat. no. ab195049), aldehyde dehydrogenase 1 (ALDH1; 1:1,000; ProteinTech Group, Inc.; cat. no. 60171-1-Ig), octamer binding protein 4 (OCT4; 1:1,000; ProteinTech Group, Inc.; cat. no. 60242-1-Ig), $\gamma$-H2AX (1:500; Abcam; cat. no. ab2893), H2AX (1:1,000; Abcam; cat. no. ab124781) and $\beta$-actin (1:5,000; ProteinTech Group, Inc.; cat. no. 60008-1-Ig). Following the primary incubation at $4^{\circ} \mathrm{C}$ for $16 \mathrm{~h}$, membranes were incubated with HRP-conjugated anti-Rabbit IgG (1:5,000; ProteinTech Group, Inc.; cat. no. SA00001-2) and anti-Mouse IgG (1:5,000; ProteinTech Group, Inc.; cat. no. SA00001-1) secondary antibodies at $37^{\circ} \mathrm{C}$ for $1 \mathrm{~h}$. Protein bands were visualized using the ECL Plus kit (cat. no. PI80196X3, Thermo Fisher Scientific, Inc.), according to the manufacturer's protocol.

In vivo tumor xenograft study. Male BALB/c nude mice aged 4-5 weeks were adopted for the construction of a subcutaneous transplantation tumor model ( $\mathrm{n}=5 /$ group). The dorsal flank of the mice were inoculated with glioma cells $\left(5 \times 10^{6} / 100 \mu \mathrm{l}\right)$. Following tumor development, tumor diameters $(<1 \mathrm{~cm})$ were detected using digital calipers and tumor volumes were calculated using the following formula: Length $\mathrm{x}$ width ${ }^{2} \mathrm{x} 0.5$, as previously described (23). Animal health and behavior, and tumor volume were monitored every 2 days. Tumors were irradiated with 2 Gy/day for 5 consecutive days when they reached $\sim 50-100 \mathrm{~mm}^{3}$, and tumor growth was recorded during the observation. All mice were euthanized 60 days after cell inoculation via intraperitoneal injection of $200 \mathrm{mg} / \mathrm{kg}$ pentobarbital sodium. All animal experiment were approved by The Institutional Animal Ethical Committee of Fujian Medical University (Fuzhou, China; approval no. 2018102). The welfare of animals in the present study was protected, and standard protocols were performed to minimize suffering.

Dual-luciferase reporter assay. The synthesized TPTEP1 or MAPK14 3'-untranslated region (UTR) wild type (WT)/mutant (Mut) sequence that harbors binding sites was subcloned into the pmirGLO dual-luciferase vector (Promega Corporation) to form pmirGLOTPTEP1/MAPK14 3'-UTR-WT or pmirGLO-TPTEP1/MAPK14 3'-UTR-Mut, which was subsequently used for co-transfection in glioma cells with mimics, inhibitor or the control sequence using Lipofectamine ${ }^{\mathrm{TM}} 2000$ reagent (Invitrogen; Thermo Fisher Scientific, Inc.). After cell transfection (48 h), Firefly and Renilla luciferase activities were detected using a Dual-Luciferase Reporter Assay System (Promega Corporation), according to the manufacturer's protocol. Firefly luciferase activity was normalized to Renilla luciferase.
RNA immunoprecipitation (RIP) assay. The Magna RNA-Binding Protein Immunoprecipitation kit (cat. no. 03-110, Millipore; Merck KGaA) was used to perform RIP assays according to the manufacturer's protocol. Cells $\left(2 \times 10^{7}\right)$ were initially scraped in a cell culture dish, followed by lysis with RIP lysis buffer. The magnetic beads (Invitrogen; Thermo Fisher Scientific, Inc.) were applied for incubation of cell extracts and conjugated with 5 ug anti-IgG antibody (cat.no. ab172730; Abcam) or anti- Agonaute 2 (Ago2) antibody (1:200; cat. no. 03-110; Millipore; Merck KGaA) supplied with the kit at $4^{\circ} \mathrm{C}$ for $16 \mathrm{~h}$. Samples were subsequently treated with Proteinase K supplied with the kit and assayed via RT-qPCR.

Bioinformatics analysis. Bioinformatics analysis was performed on the extracted mRNA-Seq data of brain lower grade glioma (LGG) and glioblastoma multiforme (GBM). The glioma expression profiles were assessed using data from The Cancer Genome Atlas (TCGA) database (https://www. cancer.gov/tcga). Accordingly, patients with lncRNA expression values lower than the median value were classified into the low expression group $(n=257)$, while the residual patients were classified into the high expression group $(n=257)$. The Gene Expression Profiling Interactive Analysis database (http://gepia.cancer-pku.cn) (24) was used to assess the association between TPTEP1 expression and overall survival time of the patients. The impact of TPTEP1 on signaling pathways was assessed via gene set enrichment analyses (GSEA). Bioinformatics tools (miRcode: http://www.mircode. org/ and RNAhybrid: https://bibiserv.cebitec.uni-bielefeld. de/rnahybrid?id=rnahybrid_view_submission) $(25,26)$ were applied to identify the miRNA that potentially interacts with TPTEP1.

Patient tissues. A total of 177 paraffin-embedded glioma samples were collected from departments of Neurology, The Second Affiliated Hospital of Fujian Medical University, (Quzhou, China). The medical records of these patients were used for collecting clinical data, including age, sex, World Health Organization (WHO) grade, recurrence status, and survival time. There are 111 male and 66 female patients, and the median age is 41 years old. All specimens were pathologically diagnosed as glioma from 2008.2-2011.10. The follow-up period ranged from 6-9.4 years. All experiments were strictly performed according to the guidelines approved by the Institutional Review Board (approval no. 2019-075), and written informed consent was provided by all patients prior to the study start.

In situ hybridization (ISH). The paraffin-embedded samples were subjected to ISH analysis. Briefly, sections were deparaffinized, rehydrated and subsequently treated with proteinase K. Following incubation with proteinase $\mathrm{K}$ at $37^{\circ} \mathrm{C}$ for $15 \mathrm{~min}$, the sections were washed with glycine once and PBS twice, and prehybridized with TPTEP1 or miR-106-5p digoxygenin-labeled probes constructed by BersinBio Corporation (http://www.bersinbio.com/). Samples were rinsed and incubated with anti-digoxygenin-HRP Fab fragments (1:200; cat. no. 200-032-156; Jackson ImmunoResearch Laboratories, Inc.) for $1 \mathrm{~h}$ at room temperature, prior to addition of DAB substrate (cat. no. DAB-0031; Fuzhou Maixin 

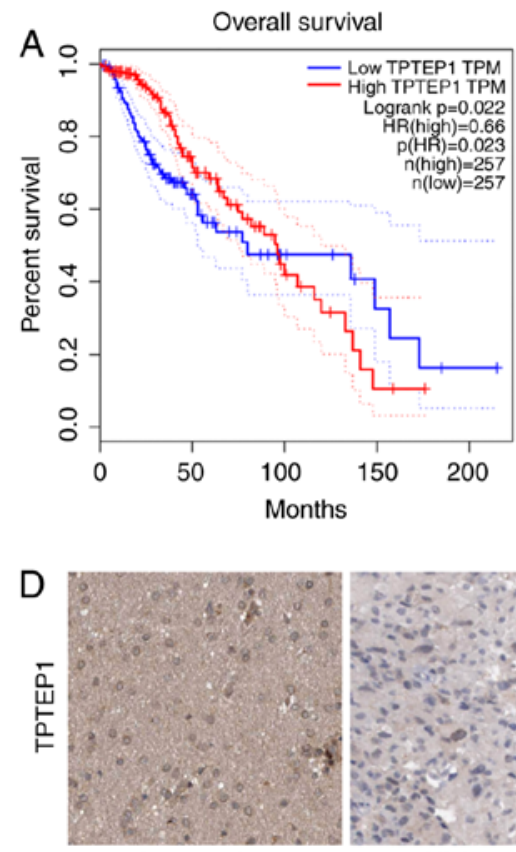

Low grade
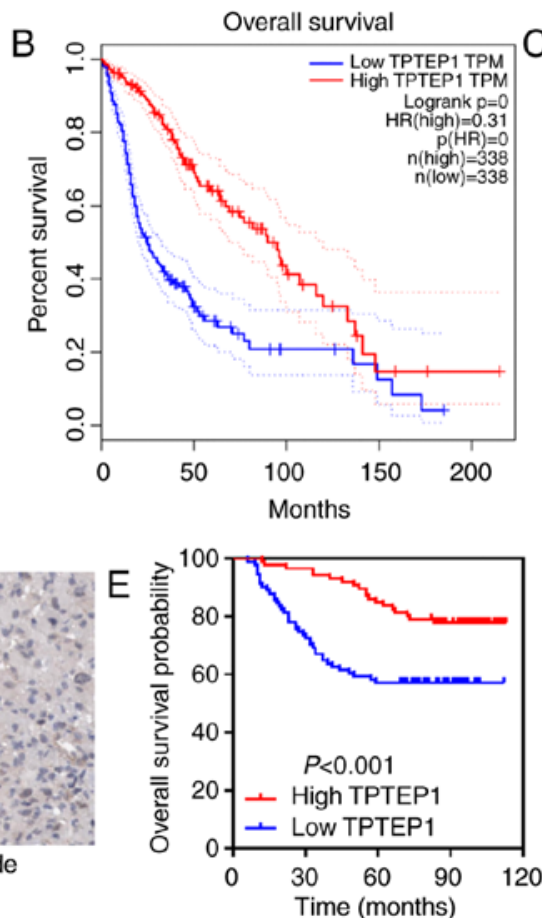

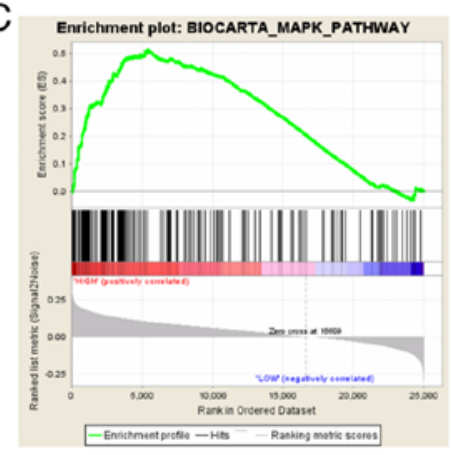

ES: 0.513 ; NES: $2.074 ;$ FDR $q=0.0$



Figure 1. TPTEP1 is downregulated in high-grade glioma and predicts a favorable patient prognosis. (A) Survival analyses for patients with glioma, with low and high TPTEP1 expression levels were performed in TCGA LGG dataset. (B) Survival analyses for patients with glioma, with low and high TPTEP1 expression levels were performed in TCGA LGG and glioblastoma multiforme datasets. (C) Gene set enrichment analyses were performed to demonstrate the involvement of TPTEP1 in the MAPK signaling pathway. (D) TPTEP1 staining in low-grade and high-grade glioma by ISH (200 x). (E) Overall survival and progression-free survival analysis were conducted in patients with glioma according to TPTEP1 expression detected by ISH (n=177). TPTEP1, transmembrane phosphatase with tensin homology pseudogene 1; TCGA, The Cancer Genome Atlas; LGG, brain lower grade glioma; ISH, in situ hybridization.

Biotech Co., Ltd.) for signal detection. Staining intensity was assessed, as previously described (7).

Immunohistochemistry (IHC). Tissues were fixed in $10 \%$ neutral formalin overnight at room temperature and dehydrated to make wax blocks. Paraffin sections $(3 \mu \mathrm{m})$ were deparaffinized, dehydrated and subsequently subjected to antigen retrieval using citrate buffer. Deparaffinized sections were blocked with $10 \%$ goat serum (Fuzhou Maixin Biotech Co., Ltd.) for $15 \mathrm{~min}$ at room temperature, and incubated with $3 \% \mathrm{H}_{2} \mathrm{O}_{2}$ for $15 \mathrm{~min}$ at room temperature to inhibit endogenous peroxidase activity and non-specific antigens. Sections were incubated with anti-p38 MAPK (1:250; ProteinTech Group, Inc.; cat. no. 66234-1-Ig) at $4^{\circ} \mathrm{C}$ for $16 \mathrm{~h}$ and HRP-conjugated secondary antibody (1:200; ProteinTech Group, Inc.; cat. no. SA00001-1) for $30 \mathrm{~min}$ at room temperature. DAB substrate was applied to assess signal detection and observed under a light microscope (x400 magnification). Staining intensity was assessed, as previously described (27).

Statistical analysis. Statistical analysis was performed using SPSS 21.0 software (IBM Corp.) and data are presented as the mean \pm standard deviation. Unpaired student's t-test was used to assess differences between two groups, while one-way ANOVA followed by Student-Newman-Keuls post hoc test was used to assess differences between multiple groups. Spearman's rank correlation test was used to measure the correlation between genes expression. The general linear model was applied for repeated measures variance analysis in assessing differences between tumor growth. The $\chi^{2}$ test was used to assess the association between gene expression and clinical characteristics. Survival analyses were performed using the Kaplan-Meier method. The Cox regression model was applied for univariate and multivariate analyses. $\mathrm{P}<0.05$ was considered to indicate a statistically significant difference.

\section{Results}

TPTEP1 is downregulated in glioma and is associated with good patient survival. TCGA data analyses were performed to determine the function of lncRNAs in glioma. TPTEP1 was identified as a potential tumor suppressor that conferred good patient prognosis (Fig. 1A and B). Relying on data from TCGA datasets, GSEA suggested that TPTEP1 participated in the modulation of the MAPK signaling pathway (Fig. 1C).

ISH was performed to detect TPTEP1 levels in 177 patients with glioma. TPTEP1 expression levels were downregulated in high-grade glioma tissues compared with low-grade glioma tissues (Fig. 1D). The clinicopathological characteristic of patients with glioma are listed in Table I. Association analysis revealed that low TPTEP1 expression levels were associated with advanced WHO grade and cancer recurrence, but not with other parameters (Table I). Consistently, Kaplan-Meier analysis revealed that patients with glioma who had high TPTEP1 expression levels had a prolonged overall survival time compared with those who had low TPTEP1 expression levels $(\mathrm{P}<0.001, \log$-rank test). In addition, patients with glioma with high TPTEP1 expression levels had a prolonged progression-free survival time compared with those with low TPTEP1 expression levels ( $\mathrm{P}=0.004$; Fig. 1E, log-rank test). 
Table I. Association between TPTEP1 expression and clinicopathological characteristics of patients with glioma (n=117).

\begin{tabular}{|c|c|c|c|c|}
\hline \multirow[b]{2}{*}{ Characteristic } & \multirow[b]{2}{*}{ Patient number, $\mathrm{n}$} & \multicolumn{2}{|c|}{ TPTEP1 expression } & \multirow[b]{2}{*}{ P-value } \\
\hline & & High, \% & Low, \% & \\
\hline \multicolumn{5}{|l|}{ Age, years } \\
\hline$\leq$ Median & 90 & $41(45.6)$ & $49(54.4)$ & 0.955 \\
\hline$>$ Median & 87 & $40(46.0)$ & $47(54.0)$ & \\
\hline \multicolumn{5}{|l|}{ Sex } \\
\hline Male & 111 & $49(44.1)$ & $62(55.9)$ & 0.575 \\
\hline Female & 66 & $32(48.5)$ & $34(51.5)$ & \\
\hline \multicolumn{5}{|l|}{ WHO grade } \\
\hline I-II & 105 & $55(52.4)$ & $50(47.6)$ & 0.033 \\
\hline III-IV & 72 & $26(36.1)$ & $46(63.9)$ & \\
\hline \multicolumn{5}{|l|}{ Recurrence } \\
\hline No & 83 & $46(55.4)$ & 37 (44.6) & 0.015 \\
\hline Yes & 94 & $35(37.2)$ & $59(62.8)$ & \\
\hline
\end{tabular}

TPTEP1, transmembrane phosphatase with tensin homology pseudogene 1; WHO, World Health Organization.

Table II. Univariate and multivariate Cox regression survival analyses of the prognostic factors of glioma.

\begin{tabular}{|c|c|c|c|c|c|c|}
\hline \multirow[b]{3}{*}{ Clinical parameter } & \multicolumn{6}{|c|}{ Overall survival } \\
\hline & \multicolumn{3}{|c|}{ Univariate analysis } & \multicolumn{3}{|c|}{ Multivariate analysis } \\
\hline & HR & $95 \% \mathrm{CI}$ & P-value & HR & $95 \% \mathrm{CI}$ & P-value \\
\hline TPTEP1 expression (low vs. high) & 2.572 & $(1.516-4.366)$ & $<0.001$ & 2.143 & $(1.062-3.554)$ & 0.031 \\
\hline Age, years (smedian vs. >median) & 0.481 & $(0.287-0.808)$ & 0.007 & 0.590 & $(0.331-1.054)$ & 0.075 \\
\hline Sex (female vs. male) & 0.660 & $(0.389-1.119)$ & 0.118 & 0.736 & $(0.407-1.330)$ & 0.310 \\
\hline WHO grade (I-II vs. III-IV) & 0.073 & $(0.041-0.128)$ & $<0.001$ & 0.427 & $(0.238-0.821)$ & 0.010 \\
\hline Recurrence (no vs. yes) & 0.095 & $(0.056-0.161)$ & $<0.001$ & 0.485 & $(0.269-0.974)$ & 0.043 \\
\hline
\end{tabular}

TPTEP1, transmembrane phosphatase with tensin homology pseudogene 1; WHO, World Health Organization; HR, hazard ratio; CI, confidence interval.

Univariate analysis was performed to elucidate the association between clinical characteristics and overall patient survival in glioma. Low TPTEP1 expression, older patients and high WHO grades were all associated with shortened overall survival time of patients with glioma. Multivariate analysis was subsequently performed to determine the independent characteristics. The results demonstrated that TPTEP1 expression and WHO grade were independent prognostic indicators for patients with glioma (Table II).

TPTEP1 suppresses glioma cell stemness and radioresistance both in vitro and in vivo. To determine the impact of TPTEP1 on glioma cell stemness and radioresistance, TPTEP1 expression levels were detected in SHG44 and U251 cells, and stable/transient TPTEP1-silenced SHG44 cells and TPTEP1-overexpressed U251 cells were established using lentiviral vectors (Fig. $2 \mathrm{~A}$ and $\mathrm{B}$ ). Both in vitro and in vivo experiments were performed to assess the stemness and radioresistance of the cell lines. TPTEP1 knockdown augmented cells stemness and radioresistance in SHG44 (Fig. 2C-F). In addition, TPTEP1 knockdown promoted cells stemness and expression of radioresistance-associated genes, including OCT4, ALDH1 and $\gamma$-H2AX (Fig. 2G). To further verify the effect of TPTEP1 on glioma cell radioresistance, mice were implanted with TPTEP1-silenced SHG44 cells and the corresponding control cells. After developing to the indicated volumes, the tumors were irradiated with 2 Gy/day for 5 consecutive days. The radiation treatment inhibited the growth of control tumors and inhibition of TPTEP1 led to resistance to radiation treatment (Fig. 2H). However, TPTEP1 overexpression induced the opposite effects in U251 cells (Fig. 2C-G).

TPTEP1 interacts with miR-106a-5p in glioma cells. Previous studies have verified the function of lncRNAs as competing endogenous RNAs (ceRNAs) in protecting miRNAs-targeted 

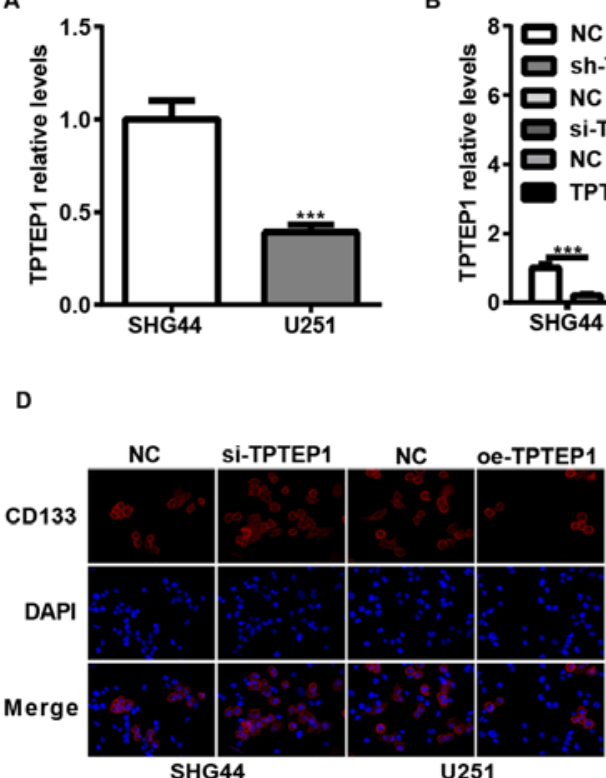

F

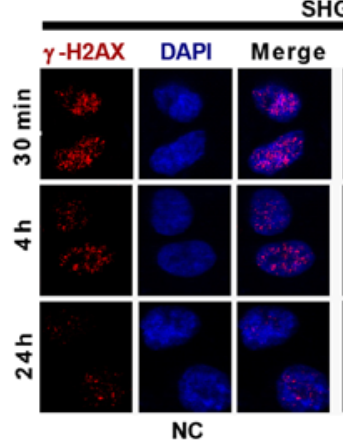

SHG44

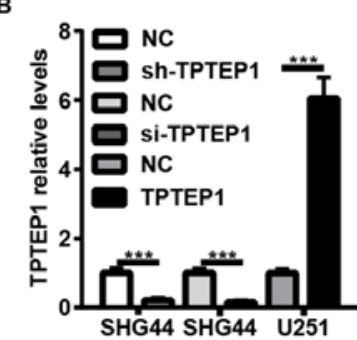

U25

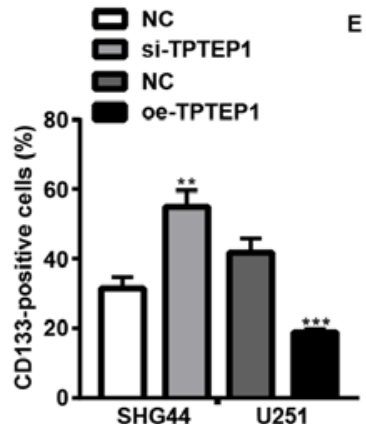

- si-TPTEP1 $\rightarrow$ si-TPTEP1+4Gy * oe-TPTEP1- oe-TPTEP1+4Gy

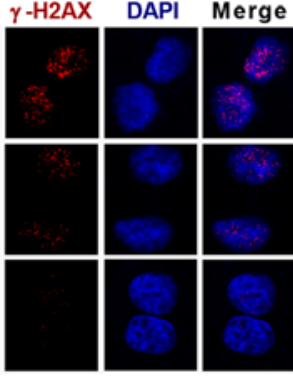

SI-TPTEP1
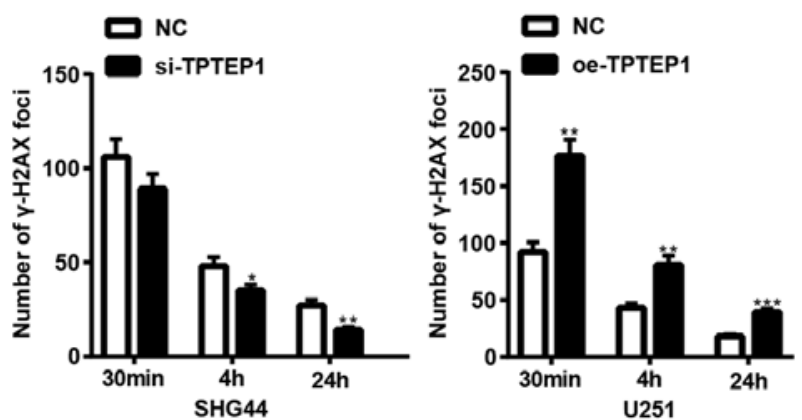

$\mathrm{E} \rightarrow \mathrm{NC} \quad \rightarrow \mathrm{NC}+4 \mathrm{~Gy}$
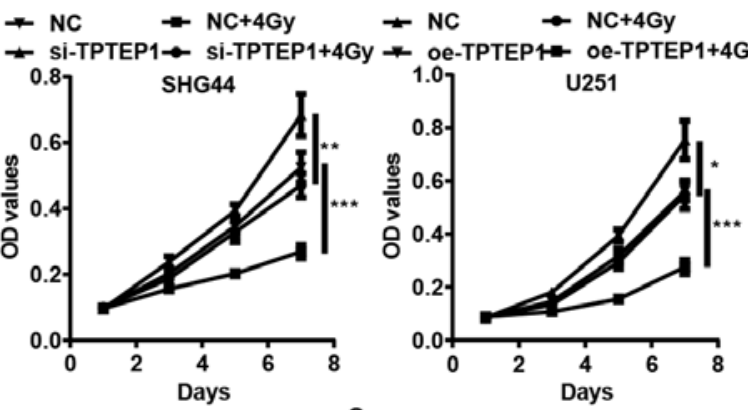

U251

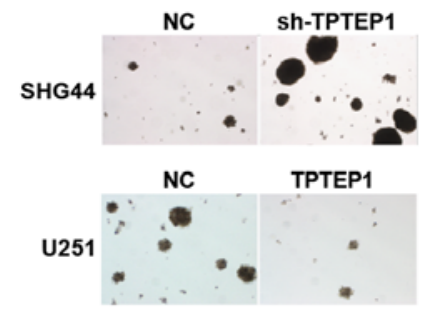

Dh-TPTEP1
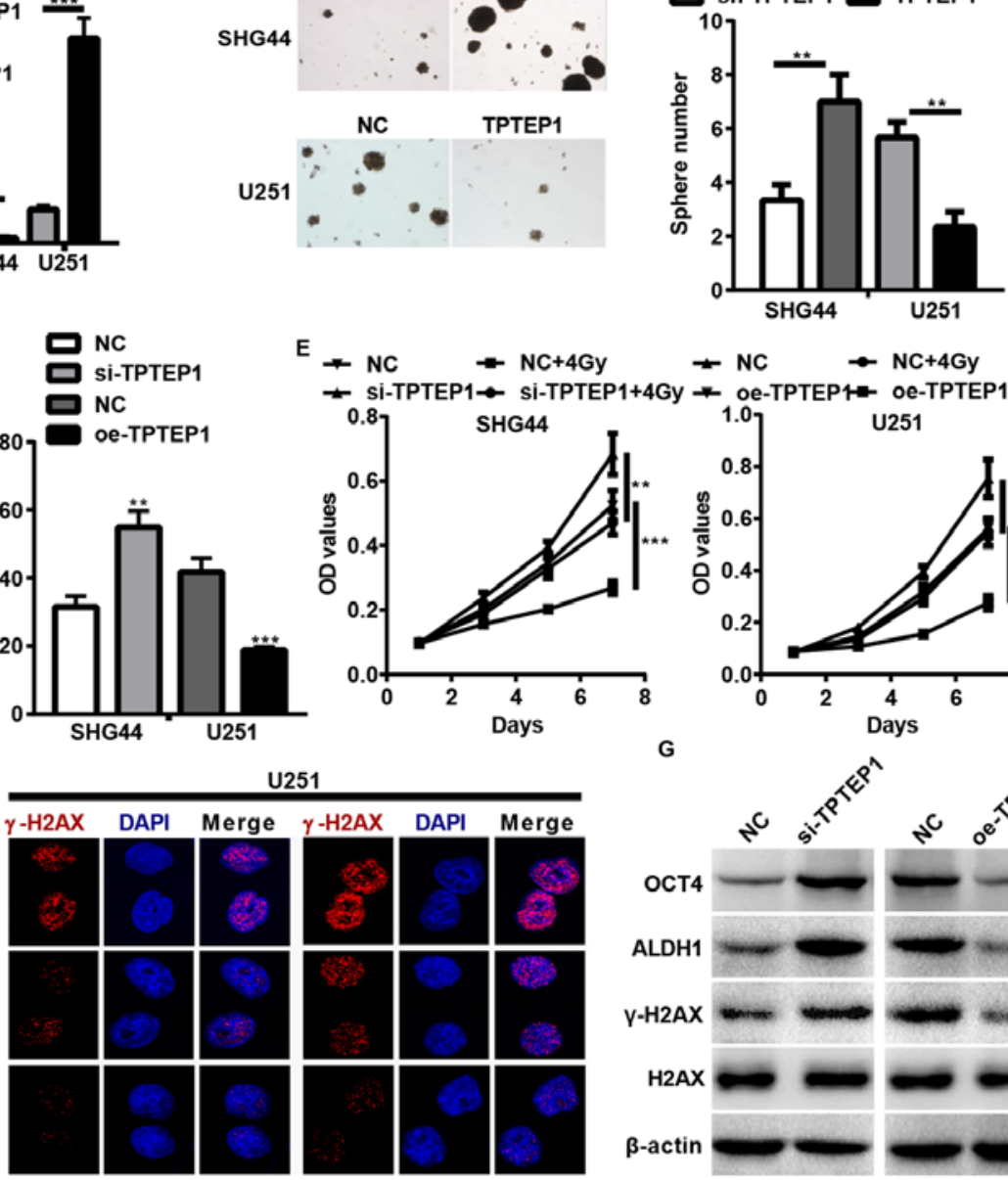

oe-TPTEP1
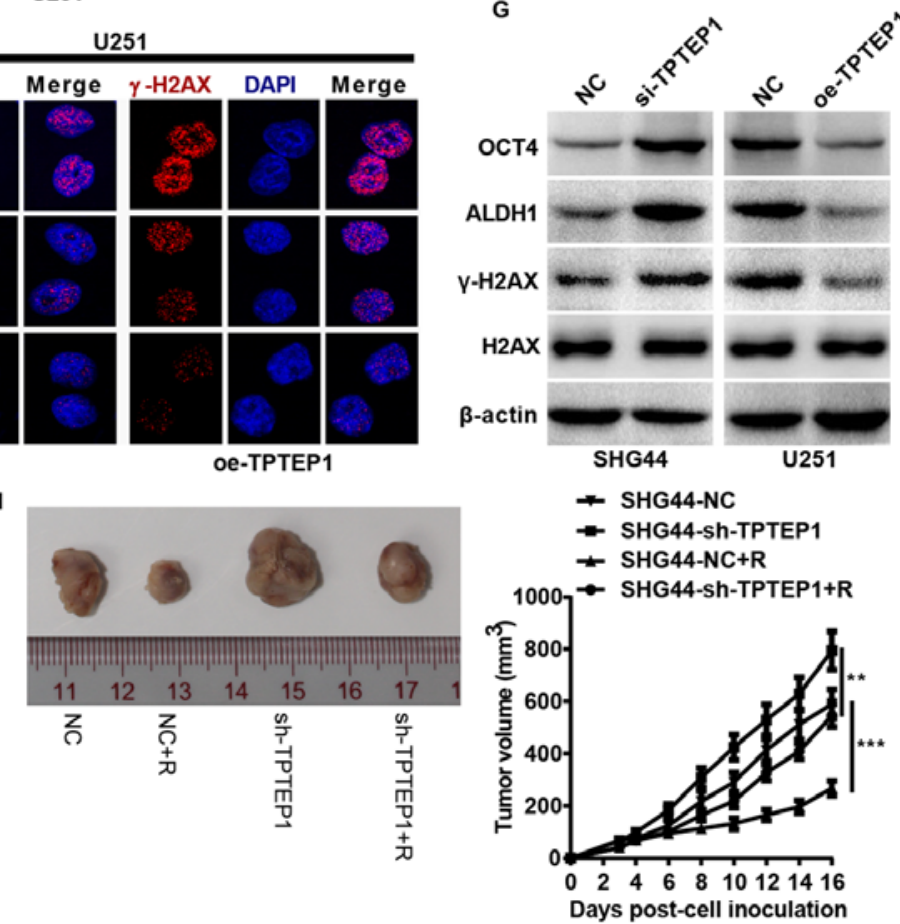

Figure 2. TPTEP1 suppresses glioma cell stemness and radioresistance in vitro and in vivo. RT-qPCR analysis was performed to detect TPTEP1 expression levels in (A) SHG44 and U251 cells and in (B) stable/transient TPTEP1-depleted SHG44, stable TPTEP1-overexpressed U251 cells and the control cells. (C) Tumorspheres formation assay was performed in stable TPTEP1-depleted SHG44 cells, stable TPTEP1-overexpressed U251 cells and the control cells (x100 magnification). (D) Immunofluorescence analysis was performed to detect the expression and location of CD133 in transient TPTEP1-depleted SHG44 cells, transient TPTEP1-overexpressed U251 cells and their corresponding controls (x400 magnification). (E) Cell proliferation and radiosensitivity assays were performed in transient TPTEP1-depleted SHG44 cells, transient TPTEP1-overexpressed U251 cells and their corresponding controls. (F) Immunofluorescence analysis was performed to evaluate $\gamma$-H2AX foci formation in transient TPTEP1-silenced SHG44 cells, transient TPTEP1-overexpressed U251 cells and their corresponding controls. (G) Western blot analysis was performed to detect OCT4, ALDH1, $\gamma$-H2AX and H2AX expression levels in transient TPTEP1-silenced SHG44 cells, transient TPTEP1-overexpressed U251 cells and their corresponding controls. (H) A subcutaneous transplantation tumor model was applied to assess glioma cell proliferation regulated by TPTEP1 and $\mathrm{R}$. ${ }^{*} \mathrm{P}<0.05,{ }^{* *} \mathrm{P}<0.01,{ }^{* * * *} \mathrm{P}<0.001$. TPTEP1, transmembrane phosphatase with tensin homology pseudogene 1; RT-qPCR, reverse transcription-quantitative PCR; OCT4, Octamer binding protein 4; ALDH1, aldehyde dehydrogenase 1; $\gamma$-H2AX, Histone $\mathrm{H} 2 \mathrm{AX} ; \mathrm{R}$, radiation.

mRNAs $(28,29)$. Thus, bioinformatics tools were applied to identify the miRNA that potentially interacts with TPTEP1. Considering base-pair conservation, expression relevance from TCGA database and reported functions of miRNAs in the regulation of CSC properties, miR-106a-5p was the focus for further study (Fig. 3A and B). Furthermore, the expression levels of TPTEP1 and miR-106a were comparable in TCGA database (Fig. 3B). The miR-106a-5p-overexpressed SHG44 cells and miR-106a-5p-silenced U251 cells were established using miR-106a-5p mimics and inhibitor, respectively 


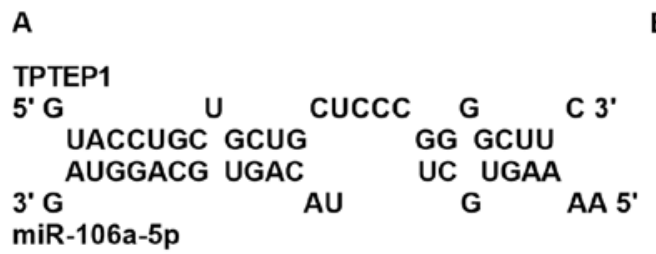

D

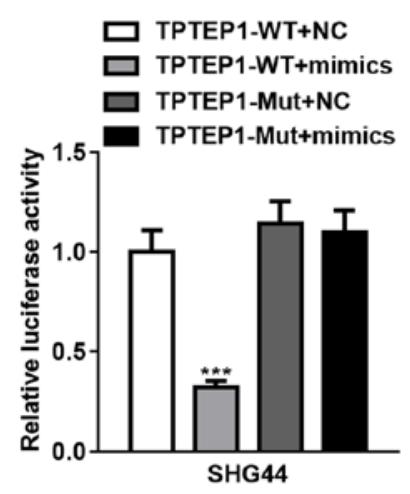

$\mathbf{F}$

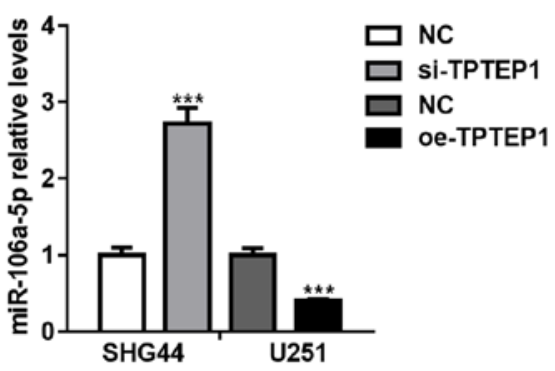

B
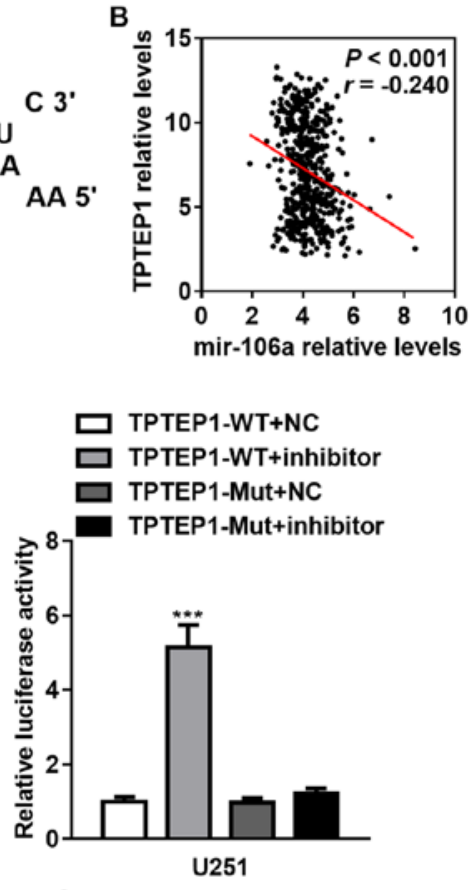

G
C

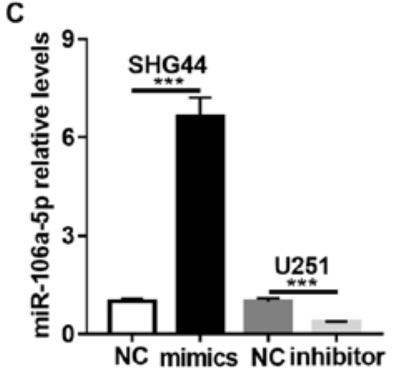

E

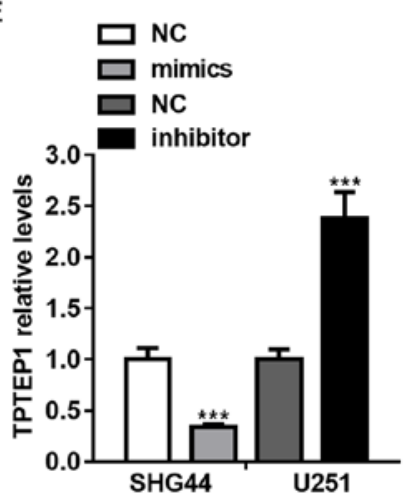

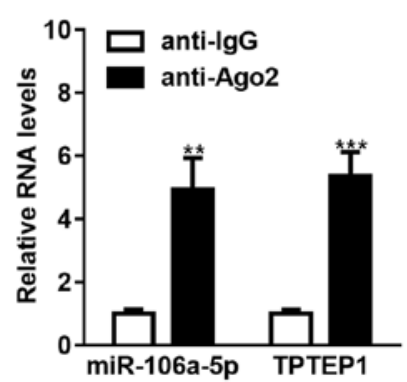

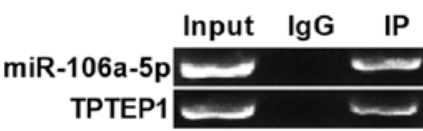

Figure 3. TPTEP1 interacts with miR-106a-5p in glioma cells. (A) miR-106a-5p and its binding sequences in TPTEP1. (B) The inverse correlation between TPTEP1 and miR-106a expression levels in TCGA LGG and glioblastoma multiforme datasets. (C) RT-qPCR analysis was performed to detect miR-106a expression levels in miR-106a-overexpressed SHG44 cells, miR-106a-5p-silenced U251 cells and the control cells. (D) Dual-luciferase reporter assay was performed to determine the interplay between miR-106a-5p and TPTEP1 in SHG44 and U251 cells. RT-qPCR analysis was performed to investigate (E) TPTEP1 expression mediated by miR-106a-5p and (F) miR-106a-5p expression mediated by TPTEP1. (G) RNA immunoprecipitation assay was performed to determine the interplay between the Ago2-recruited miR-106a-5p and TPTEP1 in U251 cells. ${ }^{* * *} \mathrm{P}<0.01,{ }^{* * * *} \mathrm{P}<0.001 \mathrm{TPTEP} 1$, transmembrane phosphatase with tensin homology pseudogene 1; miR, microRNA; TCGA, The Cancer Genome Atlas; LGG, brain lower grade glioma; RT-qPCR, reverse transcription-quantitative PCR; Ago2, Agonaute 2.

(Fig. 3C). A dual-luciferase reporter assay was performed to determine whether TPTEP1 is targeted by miR-106a-5p, and luciferase activities were downregulated by miR-106a-5p overexpression and upregulated by miR-106a-5p knockdown in SHG44 and U251 cells treated with WT TPTEP1. In glioma cells transfected with Mut TPTEP1 harboring mutations in the miR-106a-5p binding sequence inside TPTEP1, these impacts on luciferase activities were abrogated (Fig. 3D).

RT-qPCR analysis demonstrated that depletion of miR-106a-5p promoted the expression levels of TPTEP1, while upregulation of miR-106a-5p inhibited the expression levels of TPTEP1 (Fig. 3E). The impact of TPTEP1 on the expression levels of miR-106a-5p was subsequently investigated. TPTEP1 knockdown elevated miR-106a-5p expression levels, and TPTEP1 overexpression induced the opposite effect (Fig. 3F). Furthermore, TPTEP1 and miR-106a-5p were demonstrated to interact with RNA-induced silencing complexes (RISC)-related Ago2 by RNA immunoprecipitation assays
(Fig. 3G). Collectively, these results suggest that TPTEP1 interacts with miR-106a-5p, and both are assembled to RISC, forming a reciprocal regulatory circuit.

TPTEP1 promotes MAPK14 expression by antagonizing miR-106a-5p binding and activates the P38 MAPK signaling pathway. A previous study identified MAPK14 as the target of miR-106a-5p in the modulation of cell differentiation (30). To validate the interaction between the miR-106-5p binding sequence inside TPTEP1 and the MAPK14 3'UTR (Fig. 4A), western blot analysis and dual-luciferase reporter assay were performed. The results demonstrated that overexpression of miR-106-5p decreased MAPK14 and phosphorylated-MAPK14 expression levels, and miR-106-5p knockdown elevated MAPK14 expression levels (Fig. 4B). In addition, the luciferase activities were downregulated by miR-106-5p overexpression and were upregulated by miR-106-5p knockdown in SHG44 and U251 cells treated with WT MAPK14 3'UTR reporter vector 
A B

\section{C}

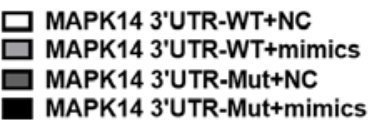

$\square$ MAPK14 3'UTR-WT+NC

MAPK14 3'UTR-WT+inhibitor MAPK14 3'UTR-Mut+NC
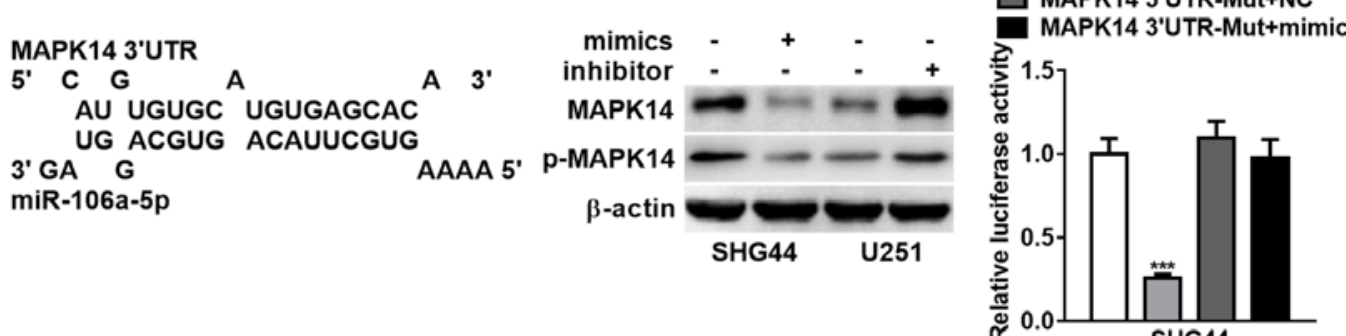

MAPK14 3'UTR-Mut+inhibitor

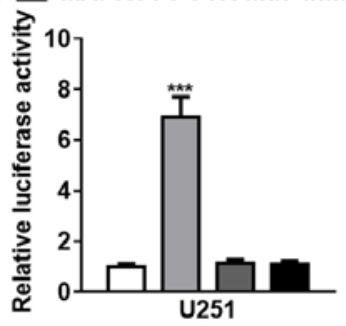

D
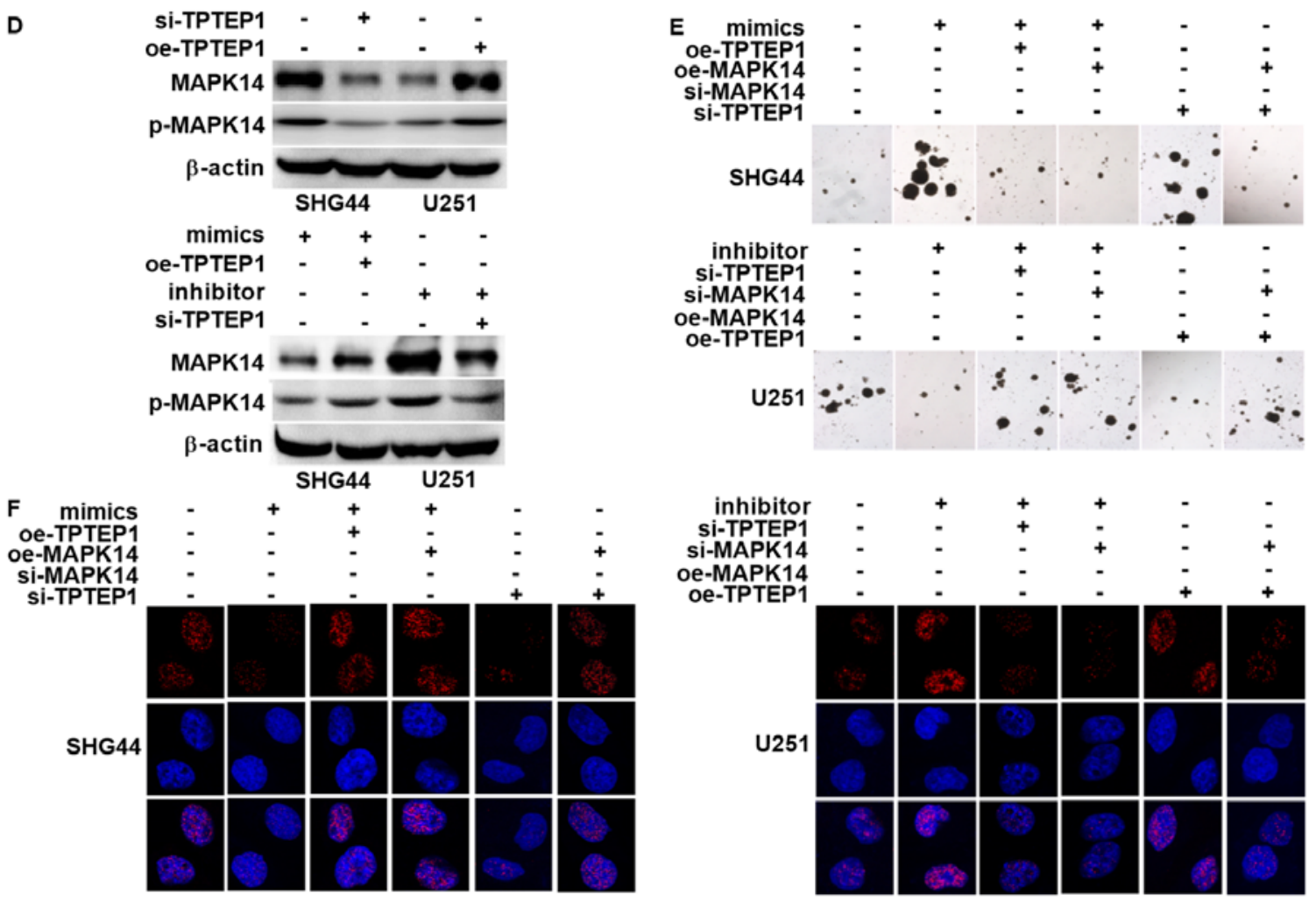

Figure 4. TPTEP1 upregulates MAPK14 by antagonizing miR-106a-5p binding and activates the P38 MAPK signaling pathway. (A) Binding sites of miR-106a-5p inside MAPK14 3'-UTR. (B) Western blot analysis was performed to detect MAPK14 and phosphorylated-MAPK14 protein expression levels in miR-106a-5p-overexpressed SHG44 cells, miR-106a-5p-silenced U251 cells and the control cells. (C) Dual-luciferase reporter assay was performed to determine the interplay between MAPK14 3'UTR and miR-106a-5p in SHG44 and U251 cells. (D) Western blot analysis was performed to detect MAPK14 and phosphorylated-MAPK14 protein expression levels in TPTEP1-depleted SHG44 cells, TPTEP1-overexpressed U251 cells, miR-106a-5p-overexpressed SHG44 cells with TPTEP1 overexpression, miR-106a-5p-silenced U251 cells with TPTEP1 knockdown and the control cells. (E) Tumorspheres formation assay was performed to assess glioma cell stemness regulated by miR-106a-5p, TPTEP1 and MAPK14. (F) Immunofluorescence analysis were performed to assess $\gamma$-H2AX foci formation regulated by miR-106a-5p, TPTEP1 and MAPK14. Sale bar $=5 \mu \mathrm{m}$. ${ }^{* * *} \mathrm{P}<0.001$. TPTEP1, transmembrane phosphatase with tensin homology pseudogene 1 ; miR, microRNA; 3'-URT, 3'-untranslated region; $\gamma$-H2AX, Histone H2AX.

(Fig. 4C). Notably, these impacts were abrogated in glioma cells transfected with Mut MAPK14 reporter vector (Fig. 4C). Taken together, these results indicate that the miR-106a-5p binding sequence within TPTEP1 interacts with the MAPK14 3'UTR, and TPTEP1 may competitively interplay with miR-106a-5p to prevent MAPK14 from repressing by miR-106a-5p.

Accordingly, the protein expression levels of MAPK14 and phosphorylated-MAPK14 were decreased in TPTEP1-silenced SHG44 cells and were increased in TPTEP1-upregulated U251 cells compared with the corresponding controls. Consistently, TPTEP1 overexpression upregulated MAPK14 and phosphorylated-MAPK14expressionlevelsinmiR-106a-5p-overexpressed SHG44 cells, while TPTEP1 knockdown downregulated MAPK14 and phosphorylated-MAPK14 expression levels in miR-106a-5p-depleted U251 cells, which suggests that TPTEP1 restores MAPK14 expression regulated by miR-106a-5p (Fig. 4D). Notably, the depletion of miR-106a-5p inhibited glioma cell stemness and radioresistance, while downregulation of TPTEP1 or MAPK14 enhanced glioma cell stemness and radioresistance in miR-106a-5p-silenced U251. However, the upregulation of TPTEP1 or MAPK14 reversed glioma cell stemness and radioresistance modulated by miR-106a-5p overexpression in SHG44, which indicates that TPTEP1 or MAPK14 may attenuate the stimulated impacts of miR-106a-5p on glioma cell stemness and radioresistance. Furthermore, overexpression of TPTEP1 was demonstrated to suppress cells stemness and radioresistance, and depletion of MAPK14 in TPTEP1-overexpressed U251 cells augmented 

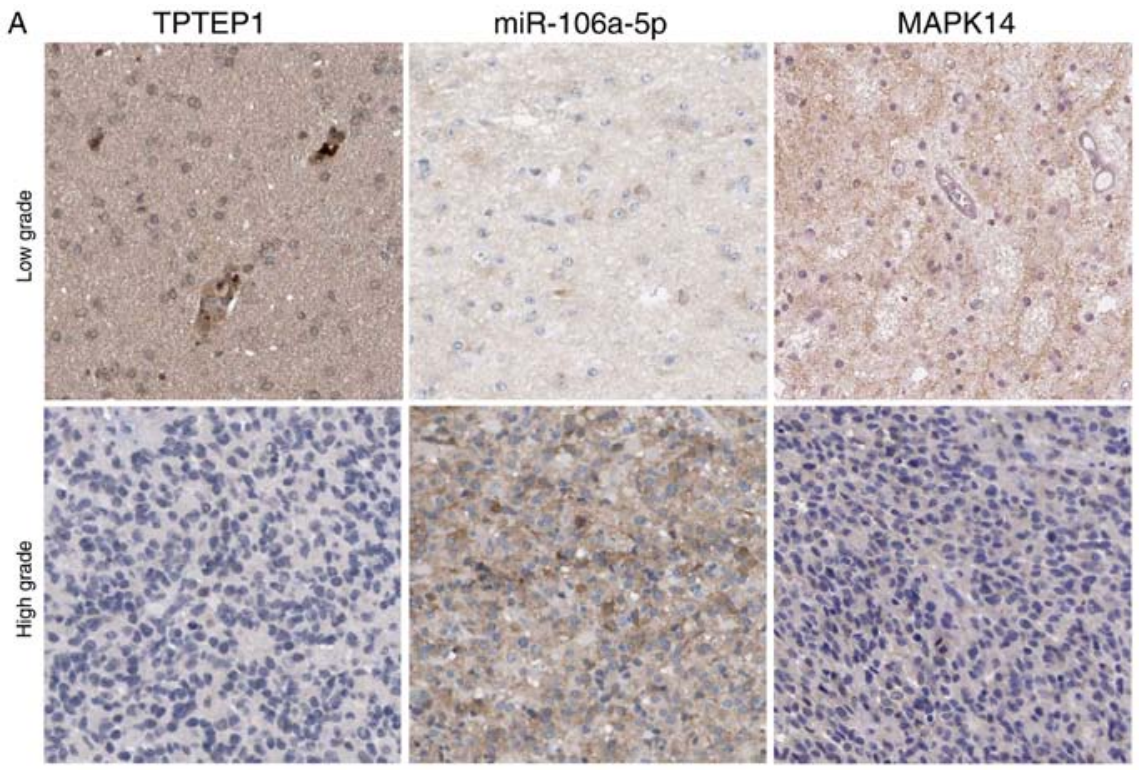

B

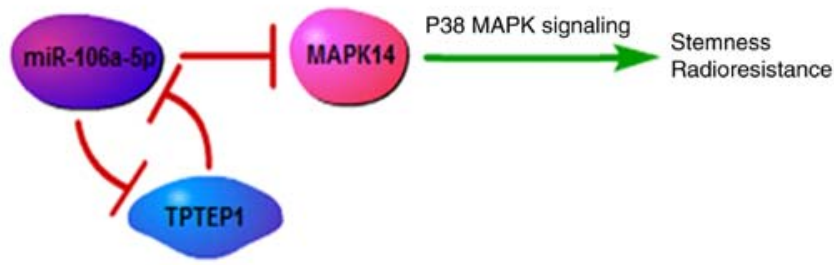

Figure 5. The TPTEP1/miR-106a-5p/MAPK14 axis in glioma. (A) In situ hybridization analysis of TPTEP1 and miR-106a-5p, and immunohistochemical staining for MAPK14 in glioma. Correlations among TPTEP1, miR-106a-5p and MAPK14 expression levels (representative images) (Spearman's rank correlation test). (B) Working model of TPTEP1/miR-106a-5p/MAPK14 axis in glioma. TPTEP1, transmembrane phosphatase with tensin homology pseudogene 1; miR, microRNA.

cell stemness and radioresistance. However, overexpression of MAPK14 reversed the impacts of TPTEP1 depletion on cells stemness and radioresistance in SHG44, which suggests that MAPK14 may reverse glioma cell stemness and radioresistance modulated by TPTEP1 (Fig. 4E and F).

Associations of TPTEP1, miR-106a-5p and MAPK14 in glioma tissues. ISH and IHC analyses were performed to detect the expression levels of miR-106a-5p and MAPK14 in the 177 glioma samples. TPTEP1 expression levels were demonstrated to be negatively associated with miR-106a-5p expression levels $(\mathrm{P}=0.013, \kappa=-0.185)$ and were positively associated with MAPK14 expression levels $(\mathrm{P}=0.032, \kappa=0.161)$ in glioma tissues. miR-106a-5p expression levels were also demonstrated to be negatively associated with MAPK14 expression levels in glioma tissues $(\mathrm{P}=0.001, \kappa=-0.253)$ (Fig. 5A). Thus, the TPTEP1/miR-106a-5p/MAPK14 axis may be responsible for regulating the suppressive effects of TPTEP1 on glioma progression (Fig. 5B).

\section{Discussion}

Increasing evidence have verified the tumor-suppressive and -promoting (oncogenic) functions of lncRNAs in different types of cancer, including lung cancer, breast cancer and brain tumor $(31,32)$. However, the function and corresponding molecular mechanism of lncRNA TPTEP1 in glioma are yet to be investigated. The results of the present study identified
TPTEP1 as a significant prognostic indicator for predicting favorable prognosis in patients with glioma. Furthermore, TPTEP1 was demonstrated to suppress stemness and radioresistance of glioma. Thus, the results of the present study suggest that mechanistically, TPTEP1 exerts its tumor-suppressive effects by triggering the P38 MAPK signaling pathway. TPTEP1 upregulates MAPK14 by antagonizing miR-106a-5p binding, and TPTEP1 interlays with miR-106a-5p to form a reciprocal regulatory circuit.

TPTEP1 acts as a tumor suppressor in hepatocellular carcinoma $(33,34)$ and lung cancer $(35)$, and has been reported to participate in the modulation of several biological functions, including cell proliferation, invasion, chemoresistance and inflammation (33-35) . In addition, bioinformatic analysis suggests that TPTEP1 may serve as a tumor suppressor in the development of GBM (36). The present study aimed to determine the properties of CSCs and investigate radioresistance modulated by lncRNA TPTEP1 in glioma, and also elucidate the tumor-suppressive role of TPTEP1 in glioma. The results demonstrated that TPTEP1 inhibited glioma cell stemness and radioresistance, thereby impeding cancer progression. TPTEP1 expression levels were downregulated in high-grade glioma tissues compared with low-grade glioma tissues. Furthermore, TPTEP1 was negatively associated with high WHO grade, recurrence status and poor patient survival time.

lncRNAs modulate gene expression through multidimensional mechanisms (37) and function as ceRNAs to prevent miRNAs from interacting with shared mRNAs (38), thus 
forming a lncRNA-miRNA-mRNA regulatory network. The present study hypothesized that the direct interaction between TPTEP1 and miR-106a-5p mutually represses their expression at the post-transcription level. The inverse association between miR-106a and TPTEP1 was validated using clinical samples. miR-106a-5p, also referred to as miR-106a, exerts an oncogenic role in different types of human cancer, including glioma $(39,40)$. In addition, miR-106a facilitates radioresistance of prostate cancer (41) and promotes cell stemness of colorectal cancer (42). The results of the present study confirmed that miRNA-106a-5p serves as an oncogene by augmenting TPTEP1-mediated stemness and radioresistance of glioma. Previous studies have demonstrated the stimulating effects of TPTEP1 and the inhibitory effect of miRNA-106a-5p on cancer cell apoptosis $(33,35,43)$. Thus, prospective studies will aim to elucidate the effect of TPTEP1 and miR-106-5p on glioma cell apoptosis.

P38 MAPK family members have dual roles in a cell type-specific and cell context-specific manner, and transduce signals that modulate cell differentiation, proliferation, migration and survival (44). Upregulating p38-MAPK signaling primes resistant GBM cells to apoptosis, and activation of $\mathrm{p} 38$ MAPK in GSCs leads to decreased neurosphere formation capacity and stem cell marker expression, which suggests that decreased stemness and radioresistance is attributed to activation of the P38 MAPK signaling pathway in most cases of glioma (45-49). The results of the present study demonstrated that MAPK14, namely P38, serves as a tumor suppressor in the development of glioma. MAPK14 exerted its inhibitory activity by antagonizing stemness and radioresistance of glioma. In addition, MAPK14 was verified as a target gene of miR-106a-5p, and cooperated with miR-106a-5p and TPTEP1 to regulate glioma progression. The rescue experiments and association analysis in tissues further confirmed the modulation of the TPTEP1/miR-106a-5p/MAPK14 axis in glioma progression. Notably, this study also has some limitations. For example, the effect of TPTEP1 and miR-106-5p on glioma cell apoptosis needs to be further studied. Additionally, the impact of TPTEP1 on tumorigenesis needs to be investigated further since this study focused on glioma progression.

Taken together, the results of the present study demonstrated that TPTEP1 functioned as a tumor suppressor that antagonized stemness and radioresistance of glioma by competitively inhibiting the interaction between miR-106a-5p and MAPK14. TPTEP1 and miR-106a-5p formed a reciprocal regulatory circuit to facilitate P38 MAPK signaling activation in glioma. Collectively, the results suggest that TPTEP1 may serve as a diagnostic and prognostic indicator for glioma, and be used as an anti-cancer target for glioma treatment.

\section{Acknowledgements}

Not applicable.

\section{Funding}

The present study was funded by the Talent Training Project of the Fujian Provincial Health and Family Planning Commission (grant no. 2017-2-29) and the Quanzhou Science and Technology Project [grant no. Z(2014)0106].

\section{Availability of data and materials}

All data generated or analyzed during this study are included in this published article.

\section{Authors' contributions}

TT acquired, analyzed and interpreted the data, and drafted the initial manuscript. RMZ designed the present study, performed the literature review, and revised and reviewed the manuscript. LXW performed the experiments and clinical studies, and MLY performed statistical analysis. All authors read and approved the final manuscript.

\section{Ethics approval and consent to participate}

All animal experiments were approved by The Institutional Animal Ethical Committee of Fujian Medical University (Fuzhou, China; approval no. 2018102). All experiments performed using human samples were approved by The institutional Ethics Committee of The Second Affiliated Hospital of Fujian Medical University (Quanzhou, China; approval no. 2019-075), and written informed consent was provided by all patients prior to the study start.

\section{Patient consent for publication}

Not applicable.

\section{Competing interests}

The authors declare that they have no competing interests.

\section{References}

1. Liu YZ, Du XX, Zhao QQ, Jiao Q and Jiang H: The expression change of OTUD3-PTEN signaling axis in glioma cells. Ann Transl Med 8: 490, 2020.

2. Larjavaara S, Mäntylä R, Salminen T, Haapasalo H, Raitanen J, Jääskeläinen J and Auvinen A: Incidence of gliomas by anatomic location. Neuro Oncol 9: 319-325, 2007.

3. Bauchet L and Ostrom QT: Epidemiology and molecular epidemiology. Neurosurg Clin N Am 30: 1-16, 2019.

4. Wang H, Xu T, Jiang Y, Xu H, Yan Y, Fu D and Chen J: The challenges and the promise of molecular targeted therapy in malignant gliomas. Neoplasia 17: 239-255, 2015.

5. Balca-Silva J, Matias D, Carmo AD, Sarmento-Ribeiro AB, Lopes MC and Moura-Neto V: Cellular and molecular mechanisms of glioblastoma malignancy: Implications in resistance and therapeutic strategies. Semin Cancer Biol 58: 130-141, 2019.

6. Visvanathan A, Patil V, Arora A, Hegde AS, Arivazhagan A, Santosh V and Somasundaram K: Essential role of METTL3-mediated $\mathrm{m}^{6} \mathrm{~A}$ modification in glioma stem-like cells maintenance and radioresistance. Oncogene 37: 522-533, 2018.

7. Lin X, Zuo S, Luo R, Li Y, Yu G, Zou Y, Zhou Y, Liu Z, Liu Y, $\mathrm{Hu}$ Y, et al: HBX-induced miR-5188 impairs FOXO1 to stimulate $\beta$-catenin nuclear translocation and promotes tumor stemness in hepatocellular carcinoma. Theranostics 9: 7583-7598, 2019.

8. Zou Y,Lin X, Bu J,LinZ, Chen Y, Qiu Y,MoH, Tang Y, Fang W and Wu Z: Timeless-stimulated miR-5188-FOXO1/ $\beta$-catenin-c-Jun feedback loop promotes stemness via ubiquitination of $\beta$-catenin in breast cancer. Mol Ther 28: 313-327, 2020.

9. Camacho CV, Choudhari R and Gadad SS: Long noncoding RNAs and cancer, an overview. Steroids 133: 93-95, 2018

10. Zhang X, Wang W, Zhu W, Dong J, Cheng Y, Yin Z and Shen F: Mechanisms and functions of long non-coding RNAs at multiple regulatory levels. Int J Mol Sci 20: 5573, 2019. 
11. Peng Z, Liu $\mathrm{C}$ and $\mathrm{Wu} \mathrm{M}$ : New insights into long noncoding RNAs and their roles in glioma. Mol Cancer 17: 61, 2018.

12. Kiang KM, Zhang XQ and Leung GK: Long non-coding RNAs: The key players in glioma pathogenesis. Cancers (Basel) 7: 1406-1424, 2015.

13. Huang X, Xiao R, Pan S, Yang X, Yuan W, Tu Z, Xu M, Zhu Y, Yin $\mathrm{Q}, \mathrm{Wu} \mathrm{Y}$, et al: Uncovering the roles of long non-coding RNAs in cancer stem cells. J Hematol Oncol 10: 62, 2017.

14. Ren J, Ding L, Zhang D, Shi G, Xu Q, Shen S, Wang Y, Wang T and Hou Y: Carcinoma-associated fibroblasts promote the stemness and chemoresistance of colorectal cancer by transferring exosomal lncRNA H19. Theranostics 8: 3932-3948, 2018.

15. Zhuang J, Shen L, Yang L, Huang X, Lu Q, Cui Y, Zheng X, Zhao X, Zhang D, Huang R, et al: TGF $\beta 1$ promotes gemcitabine resistance through regulating the LncRNA-LET/NF90/miR-145 signaling axis in bladder cancer. Theranostics 7: 3053-3067, 2017.

16. Mazor G, Levin L, Picard D, Ahmadov U, Carén H, Borkhardt A, Reifenberger G, Leprivier G, Remke M and Rotblat B: The lncRNA TP73-AS1 is linked to aggressiveness in glioblastoma and promotes temozolomide resistance in glioblastoma cancer stem cells. Cell Death Dis 10: 246, 2019.

17. Yu M, Xue Y, Zheng J, Liu X, Yu H, Liu L, Li Z and Liu Y: Linc00152 promotes malignant progression of glioma stem cells by regulating miR-103a-3p/FEZF1/CDC25A pathway. Mol Cancer 16: 110, 2017.

18. Kim SS, Harford JB, Moghe M, Rait A, Pirollo KF and Chang EH: Targeted nanocomplex carrying siRNA against MALAT1 sensitizes glioblastoma to temozolomide. Nucleic Acids Res 46: 1424-1440, 2018.

19. Dai X, Liao K, Zhuang Z, Chen B, Zhou Z, Zhou S, Lin G Zhang F, Lin Y, Miao Y, et al: AHIF promotes glioblastoma progression and radioresistance via exosomes. Int J Oncol 54: 261-270, 2019

20. Liao K, Ma X, Chen B, Lu X, Hu Y, Lin Y, Huang R and Qiu Y: Upregulated AHIF-mediated radioresistance in glioblastoma. Biochem Biophys Res Commun 509: 617-623, 2019.

21. Zheng R, Yao Q, Ren C, Liu Y, Yang H, Xie G, Du S, Yang K and Yuan Y: Upregulation of long noncoding RNA small nucleolar RNA host gene 18 promotes radioresistance of glioma by repressing semaphorin 5A. Int J Radiat Oncol Biol Phys 96: 877-887, 2016

22. Livak KJ and Schmittgen TD: Analysis of relative gene expression data using real-time quantitative PCR and the 2(-Delta Delta C(T)) method. Methods 25: 402-408, 2001.

23. Li P, Wang Q and Wang H: MicroRNA-204 inhibits the proliferation, migration and invasion of human lung cancer cells by targeting PCNA-1 and inhibits tumor growth in vivo. Int J Mol Med 43: 1149-1156, 2019.

24. Tang Z, Li C, Kang B, Gao G, Li C and Zhang Z: GEPIA: A web server for cancer and normal gene expression profiling and interactive analyses. Nucleic Acids Res 45 (W1): W98-W102, 2017.

25. Jeggari A, Marks DS and Larsson E: miRcode: A map of putative microRNA target sites in the long non-coding transcriptome. Bioinformatics 28: 2062-2063, 2012.

26. Rehmsmeier M, Steffen P, Hochsmann M and Giegerich R: Fast and effective prediction of microRNA/target duplexes. RNA 10: $1507-1517,2004$

27. Lin X, Li AM, Li YH, Luo RC, Zou YJ, Liu YY, Liu C, Xie YY, Zuo S, Liu Z, et al: Silencing MYH9 blocks HBx-induced GSK3 $\beta$ ubiquitination and degradation to inhibit tumor stemness in hepatocellular carcinoma. Signal Transduct Target Ther 5: 13, 2020.

28. Karreth FA and Pandolfi PP: ceRNA cross-talk in cancer: When ce-bling rivalries go awry. Cancer Discov 3: 1113-1121, 2013

29. Thomson DW and Dinger ME: Endogenous microRNA sponges: Evidence and controversy. Nat Rev Genet 17: 272-283, 2016.

30. Naka-Kaneda H, Nakamura S, Igarashi M, Aoi H, Kanki H, Tsuyama J, Tsutsumi S, Aburatani H, Shimazaki T and Okano H: The miR-17/106-p38 axis is a key regulator of the neurogenic-to-gliogenic transition in developing neural stem/progenitor cells. Proc Natl Acad Sci USA 111: 1604-1609, 2014

31. Bhan A, Soleimani M and Mandal SS: Long noncoding RNA and cancer: A new paradigm. Cancer Res 77: 3965-3981, 2017.
32. Zhang $Y$ and Tang L: The application of lncRNAs in cancer treatment and diagnosis. Recent Pat Anticancer Drug Discov 13: 292-301, 2018

33. Ding H, Liu J, Zou R, Cheng P and Su Y: Long non-coding RNA TPTEP1 inhibits hepatocellular carcinoma progression by suppressing STAT3 phosphorylation. J Exp Clin Cancer Res 38: 189,2019

34. Ding H, Zhang X, Su Y, Jia C and Dai C: GNAS promotes inflammation-related hepatocellular carcinoma progression by promoting STAT3 activation. Cell Mol Biol Lett 25: 8, 2020.

35. Cao F, Wang Z, Feng Y, Zhu H, Yang M, Zhang S and Wang X lncRNA TPTEP1 competitively sponges miR-328-5p to inhibit the proliferation of non-small cell lung cancer cells. Oncol Rep 43: 1606-1618, 2020.

36. Zheng J, Su Z, Kong Y, Lin Q, Liu H, Wang Y and Wang J: lncRNAs predicted to interfere with the gene regulation activity of miR-637 and miR-196a-5p in GBM. Front Oncol 10: 303, 2020.

37. Marchese FP, Raimondi I and Huarte M: The multidimensional mechanisms of long noncoding RNA function. Genome Biol 18: 206, 2017.

38. Tay Y, Rinn J and Pandolfi PP: The multilayered complexity of ceRNA crosstalk and competition. Nature 505: 344-352, 2014.

39. Wang Z, Wang B, Shi Y, Xu C, Xiao HL, Ma LN, Xu SL, Yang L, Wang QL, Dang WQ, et al: Oncogenic miR-20a and miR-106a enhance the invasiveness of human glioma stem cells by directly targeting TIMP-2. Oncogene 34: 1407-1419, 2015.

40. Zhao H, Shen J, Hodges TR, Song R, Fuller GN and Heimberger AB: Serum microRNA profiling in patients with glioblastoma: A survival analysis. Mol Cancer 16: 59, 2017.

41. Hoey C, Ray J, Jeon J, Huang X, Taeb S, Ylanko J, Andrews DW, Boutros PC and Liu SK: miRNA-106a and prostate cancer radioresistance: A novel role for LITAF in ATM regulation. Mol Oncol 12: 1324-1341, 2018.

42. Qin Y, Chen X, Liu Z, Tian X and Huo Z: miR-106a reduces 5-fluorouracil (5-FU) sensitivity of colorectal cancer by targeting dual-specificity phosphatases 2 (DUSP2). Med Sci Monit 24: 4944-4951, 2018.

43. Dong S, Zhang X and Liu D: Overexpression of long noncoding RNA GAS5 suppresses tumorigenesis and development of gastric cancer by sponging miR-106a-5p through the Akt/mTOR pathway. Biol Open 8: bio041343, 2019.

44. Wagner EF and Nebreda AR: Signal integration by JNK and p38 MAPK pathways in cancer development. Nat Rev Cancer 9: 537-549, 2009.

45. Sato A, Okada M, Shibuya K, Watanabe E, Seino S, Narita Y, Shibui S, Kayama T and Kitanaka C: Pivotal role for ROS activation of p38 MAPK in the control of differentiation and tumor-initiating capacity of glioma-initiating cells. Stem Cell Res 12: 119-131, 2014

46. Wu J, Zhang H, Xu Y, Zhang J, Zhu W, Zhang Y, Chen L, Hua W and Mao Y: Juglone induces apoptosis of tumor stem-like cells through ROS-p38 pathway in glioblastoma. BMC Neurol 17: 70, 2017.

47. Liu P, Brown S, Goktug T, Channathodiyil P, Kannappan V, Hugnot JP, Guichet PO, Bian X, Armesilla AL, Darling JL and Wang W: Cytotoxic effect of disulfiram/copper on human glioblastoma cell lines and ALDH-positive cancer-stem-like cells. Br J Cancer 107: 1488-1497, 2012.

48. Tamura K, Wakimoto H, Agarwal AS, Rabkin SD, Bhere D, Martuza RL, Kuroda T, Kasmieh R and Shah K: Multimechanistic tumor targeted oncolytic virus overcomes resistance in brain tumors. Mol Ther 21: 68-77, 2013.

49. Yang W, Shen Y, Wei J and Liu F: MicroRNA-153/Nrf-2/GPx1 pathway regulates radiosensitivity and stemness of glioma stem cells via reactive oxygen species. Oncotarget 6: 22006-22027, 2015. International (CC BY-NC-ND 4.0) License. 